\title{
Multivariable Model Predictive Control of Thin Film Surface Roughness and Slope: Application to a 2D Kinetic Monte-Carlo Model
}

\author{
Xinyu Zhang, Gerassimos Orkoulas and Panagiotis D. Christofides
}

\begin{abstract}
This work focuses on the application of a multivariable model predictive controller that regulates thin film surface roughness and mean slope to a two-dimensional kinetic Monte-Carlo thin film growth model using both substrate temperature and deposition rate as manipulated inputs. The description of the thin film growth involving both adsorption and surface migration is first given. Surface roughness and surface slope are defined as the root-mean-squares of the surface height profile and the surface slope profile, respectively. The dynamics of the evolution of the thin film surface height profile are assumed to be described by an Edwards-Wilkinsontype equation (a second-order stochastic partial differential equation) in two spatial dimensions. Analytical solutions of the expected surface roughness and surface slope are obtained on the basis of the Edwards-Wilkinson equation and are used in the controller design. The model parameters of the EdwardsWilkinson equation depend on the substrate temperature and deposition rate. This dependence is used in the formulation of the predictive controller to predict the influence of the control action on the surface roughness and slope at the end of the growth process. The model predictive controller involves constraints on the magnitude and rate of change of the control action and optimizes a cost that involves penalty on both surface roughness and mean slope from the set-point values. The controller is applied to the two-dimensional kinetic MonteCarlo thin film growth model and is shown to successfully regulate surface roughness and mean slope to set-point values at the end of the deposition.
\end{abstract}

Key words: Surface mean slope, surface roughness, light trapping optimization, model predictive control, distributed parameter systems, thin film growth.

\section{INTRODUCTION}

Over the last decade, significant research efforts have demonstrated (e.g., [19], [12], [10], [14], [13]) that the surface morphology of thin films, in particular the thin film surface roughness and the mean surface slope (root-meansquare (rms) of the surface slope profile), strongly influence the light trapping efficiency of thin-film solar cells. This motivates designing model-based control systems with the objective of tailoring thin film surface morphology to desired characteristics to improve light trapping efficiency.

With respect to previous work on modeling and control of thin film growth, microscopic/multiscale modeling approaches have been developed and used to design feedback control systems for thin film surface roughness, porosity

Xinyu Zhang, Gerassimos Orkoulas and Panagiotis D. Christofides are with the Department of Chemical and Biomolecular Engineering, University of California, Los Angeles, CA 90095-1592, USA. Panagiotis D. Christofides is also with the Department of Electrical Engineering, University of California, Los Angeles, CA 90095-1592, USA, xinyuzhang@ucla.edu, makis@seas.ucla.edu, pdc@seas.ucla.edu. and thickness control (see, for example, [1], [15], [16], [7], [8] for representative results and references). Specifically, modeling approaches can be broadly classified into the following categories: kinetic Monte Carlo (kMC) methods and stochastic differential equation (SDE) models. Since kMC models are not available in closed form, they cannot be readily used for feedback control design and system-level analysis. On the other hand, SDE models possess a closed form that enables their use as the basis for the design of feedback controllers which can regulate thin film surface morphology and microstructure. Recently, we initiated an effort towards modeling and control of surface mean slope which strongly influences the light reflectance and transmittance properties of thin films. In this direction, we have studied dynamics and lattice size dependence of surface mean slope [9] and designed predictive control algorithms for both surface roughness and slope regulation using stochastic partial differential equations (PDEs) in one spatial dimension [22].

In a previous work [21], we focused on the development of a model predictive controller to regulate both surface roughness and slope and applied this controller to a twodimensional (2D) Edwards-Wilkinson-type equation. In the present paper, we focus on the application of the multivariable model predictive controller of [21] to a twodimensional kinetic Monte-Carlo thin film growth model to regulate thin film surface roughness and mean slope at appropriate set-points, using both substrate temperature and deposition rate as manipulated inputs. The dynamics of the evolution of the thin film surface height profile are assumed to be described by an Edwards-Wilkinson-type equation in two spatial dimensions. The model parameters of the Edwards-Wilkinson (EW) equation depend on the substrate temperature and deposition rate and are computed on the basis of open-loop data obtained from the two-dimensional kinetic Monte-Carlo simulator. This dependence is used in the formulation of an EW-based predictive controller that involves constraints on the magnitude and rate of change of the control action and optimizes a cost that involves penalty on both surface roughness and mean slope from the set-point values. The controller is successfully applied to the twodimensional kinetic Monte-Carlo thin film growth model.

\section{Preliminaries}

\section{A. Description of thin film deposition process}

In this work, we focus on a thin film deposition process involving deposition and surface migration in two-dimensions (2D). We make the solid-on-solid (SOS) assumption during 
the growth, where particles land on top of the existing surface particles. Periodic boundary conditions are applied in the directions that are perpendicular to the growth direction. Lattice size is defined as the number of sites in the lateral direction. The lattice size in both lateral directions is the same, i.e., the deposition takes place on a square lattice.

Fig. 1 shows the lattice model of the thin film deposition process. In Fig. 1, the incident particles are deposited vertically onto the thin film. The surface particles, i.e., the highest particles of the lattice sites, are subject to a migration event. Specifically, the deposition and migration events are separated into two independent microscopic events (e.g., [11]). The deposition event is a random deposition. With respect to migration, the top particle of a lattice site is subject to migration with a probability that depends on its local environment (i.e., number of nearest neighbors) and the substrate temperature. The migration rate (probability) follows an Arrhenius-type law as follows:

$$
r_{m, i}=v_{0} \exp \left(-\frac{E_{s}+n_{i} E_{n}}{k_{B} T}\right),
$$

where $r_{m, i}$ denotes the migration rate of the $i$-th surface particle, $v_{0}=2 k_{B} T / h$ is a pre-exponential factor, $n_{i}=0,1,2,3$, is the number of the nearest neighbors in the same layer of the surface particle on the $i$ th lattice site, $E_{s}$ and $E_{n}$ are the contribution to the activation energy barrier from the surface site and from each nearest neighbor, respectively, $k_{B}$ is Boltzmann's constant, $h$ is Planck's constant, and $T$ is the substrate temperature. The following values were used for the process parameters

$$
E_{s}=1.58 \mathrm{eV} \quad E_{n}=0.28 \mathrm{eV}
$$

When a surface particle is subject to migration, the particle moves to a neighboring site with a lower surface height. If two or more neighboring sites are lower than the surface height, the migrating particle randomly moves to one of these neighboring sites with equal probability. We note that when $n_{i}$ equals the number of nearest neighboring sites, the particle is fully surrounded by other particles and cannot move. We use the continuous-time kinetic Monte Carlo (CTMC) algorithm [17] to simulate the thin deposition process model.

\section{B. Edwards-Wilkinson Equation for Surface Height Dynam- ics}

The Edwards-Wilkinson (EW) equation has been demonstrated to adequately describe the dynamics of the evolution of the surface height profile in many thin-film growth processes that involve a thermal balance between atom adsorption and surface migration (e.g., [2], [3], [4], [9]). In this work, we use an EW-type equation in two spatial dimensions to describe the film surface evolution of the following form:

$$
\frac{\partial h}{\partial t}=c+c_{2}\left(\frac{\partial^{2} h}{\partial x^{2}}+\frac{\partial^{2} h}{\partial y^{2}}\right)+\xi(x, y, t)
$$

where $x \in[0, \pi], y \in[0, \pi]$ are the spatial coordinates, $t$ is the time, $h(x, y, t)$ is the surface height, and $\xi(x, y, t)$ is a
Fig. 1. Thin film deposition processes in the the two dimensional square lattice.

Gaussian white noise with a zero mean and the following covariance:

$$
\left\langle\xi(x, y, t) \xi\left(x^{\prime}, y^{\prime}, t^{\prime}\right)\right\rangle=\sigma^{2} \delta\left(x-x^{\prime}\right) \delta\left(y-y^{\prime}\right) \delta\left(t-t^{\prime}\right)
$$

where $\delta(\cdot)$ denotes the Dirac delta function. $c, c_{2}$, and $\sigma^{2}$ are model parameters that have explicit dependence on the macroscopic operating variables, i.e., the substrate temperature, $T$, and the deposition rate, $W$. Specifically, $c$ is related to the growth rate of the average surface height and $c_{2}$ is related to the effect of surface migration. These model parameters can be identified on the basis of kinetic Monte Carlo simulation or experimental data [11], [6]; please see subsection II-D below for a detailed discussion on the computation of $c, c_{2}$ and $\sigma^{2}$. The stochastic PDE of eq (3) is subject to periodic boundary conditions (PBCs) of the form:

$$
\begin{aligned}
h(0, y, t) & =h(\pi, y, t) & h(x, 0, t) & =h(x, \pi, t) \\
\frac{\partial h}{\partial x}(0, y, t) & =\frac{\partial h}{\partial x}(\pi, y, t) & \frac{\partial h}{\partial y}(x, 0, t) & =\frac{\partial h}{\partial y}(x, \pi, t)
\end{aligned}
$$

and the initial condition

$$
h(x, y, 0)=h_{0}(x, y)
$$

To analyze the dynamics and obtain a finite-dimensional approximation of the EW equation, we first consider the eigenvalue problem of the linear operator of eq (3) subject to the periodic boundary conditions of eqs (5) and (6):

$$
\begin{gathered}
\mathscr{A} \phi_{m, n}(x, y)=c_{2}\left(\frac{\partial^{2}}{\partial x^{2}}+\frac{\partial^{2}}{\partial y^{2}}\right) \phi_{m, n}(x, y)=\lambda_{m, n} \phi_{m, n}(x, y) \\
\nabla^{j} \phi_{m, n}(0, y)=\nabla^{j} \phi_{m, n}(\pi, y), \quad j=0,1 \\
\nabla^{j} \phi_{m, n}(x, 0)=\nabla^{j} \phi_{m, n}(x, \pi), \quad j=0,1
\end{gathered}
$$

where $\lambda_{m, n}$ denotes an eigenvalue, $\phi_{m, n}$ denotes an eigenfunction, and $\nabla^{j}, j=0,1$, denotes the gradient of a given function. The solution of the eigenvalue problem is as 
follows:

$$
\begin{aligned}
\lambda_{m, n} & =4 c_{2}\left(m^{2}+n^{2}\right) \\
\phi_{1, m, n} & =\frac{2}{\pi} \sin (2 m x) \sin (2 n y) \\
\phi_{2, m, n} & = \begin{cases}\frac{1}{\pi} & m=0 \text { and } n=0 \\
\frac{2}{\pi} \cos (2 m x) \cos (2 n y) & m \neq 0 \text { and } n \neq 0 \\
\frac{\sqrt{2}}{\pi} \cos (2 m x) \cos (2 n y) & \text { otherwise }\end{cases} \\
\phi_{3, m, n} & = \begin{cases}0 & m=0 \\
\frac{2}{\pi} \sin (2 m x) \cos (2 n y) & m \neq 0, n \neq 0 \\
\frac{\sqrt{2}}{\pi} \sin (2 m x) & m \neq 0, n=0\end{cases} \\
\phi_{4, m, n} & = \begin{cases}0 & n=0 \\
\frac{2}{\pi} \cos (2 m x) \sin (2 n y) & n \neq 0, m \neq 0 \\
\frac{\sqrt{2}}{\pi} \sin (2 n y) & n \neq 0, m=0\end{cases}
\end{aligned}
$$

The solution of the EW equation of eq (3) can be expanded in an infinite series in terms of the eigenfunctions of the operator of eq (8) as follows:

$$
h(x, y, t)=\sum_{m=0}^{+\infty} \sum_{n=0}^{+\infty} \sum_{p=1}^{4} \phi_{p, m, n} z_{p, m, n},
$$

where $z_{1, m, n}, z_{2, m, n}, z_{3, m, n}$, and $z_{4, m, n}$ are time-varying coefficients.

Substituting the above expansion for the solution, $h(x, y, t)$ into eq (3) and taking the inner product with the adjoint eigenfunctions, the following system of infinite stochastic linear ordinary differential equations (ODEs) for the temporal evolution of the time-varying coefficients is obtained:

$$
\begin{gathered}
\frac{d z_{2,0,0}}{d t}=\pi c+\xi_{2,0,0}(t), \\
\frac{d z_{p, m, n}}{d t}=\lambda_{m, n} z_{p, m, n}+\xi_{p, m, n}(t) \\
p=1,2,3,4, \quad m, n=0,1, \cdots, \infty, \quad m^{2}+n^{2} \neq 0
\end{gathered}
$$

where $\xi_{p, m, n}=\int_{0}^{\pi} \int_{0}^{\pi} \xi(x, y, t) \phi_{p, m, n} d x d y$ is the projection of the noise $\xi(x, y, t)$ on the ODE for $z_{p, m, n}$. The noise term, $\xi_{p, m, n}$, has zero mean and covariance

$$
\left\langle\xi_{p, m, n}(t) \xi_{p, m, n}\left(t^{\prime}\right)\right\rangle=\sigma^{2} \delta\left(t-t^{\prime}\right)
$$

The temporal evolution of the variance of mode $z_{p, m, n}$ can be obtained from the solution of the linear ODE of eqs (17) and (18) as follows:

$$
\begin{gathered}
\left\langle z_{p, m, n}^{2}(t)\right\rangle=e^{2 \lambda_{m, n}\left(t-t_{0}\right)}\left\langle z_{p, m, n}^{2}\left(t_{0}\right)\right\rangle+\sigma^{2} \frac{e^{2 \lambda_{m, n}\left(t-t_{0}\right)}-1}{2 \lambda_{m, n}} \\
m^{2}+n^{2} \neq 0
\end{gathered}
$$

For feedback control purposes (see Section III below), the modes can be calculated from a surface height measurement as follows:

$$
z_{p, m, n}(t)=\int_{0}^{\pi} \int_{0}^{\pi} h(x, y, t) \phi_{p, m, n}(x, y) d x d y .
$$

In many circumstances, only discrete height profile measurements are available, thus eq (21) can be approximated by

$$
z_{p, m, n}(t)=\frac{\pi^{2}}{L^{2}} \sum_{i=0}^{L-1} \sum_{j=0}^{L-1} \hat{h}(i, j, t) \phi_{p, m, n}(i, j)
$$

where $L$ is the number of spatial height sampling (measurement) points in $[0, \pi]$ in both $x$ and $y$ coordinates and $\hat{h}(i, j, t)=h\left(x_{i}, y_{j}, t\right)=h\left(\frac{i \pi}{L}, \frac{j \pi}{L}, t\right)$. It is worth pointing out that, when discrete height measurements are available, the highest number of modes that can be accurately calculated is limited by the spatial sampling points, $m, n \leq L / 2$.

\section{Film Surface Roughness and rms Slope}

Thin film surface morphology can be characterized by roughness and rms slope. Roughness is defined as the rootmean-square of the surface height profile:

$$
\begin{aligned}
r(t) & =\sqrt{\frac{1}{\pi^{2}} \int_{0}^{\pi} \int_{0}^{\pi}(h(x, y, t)-\bar{h}(t))^{2} d x d y} \\
& \approx \sqrt{\frac{1}{L^{2}} \sum_{i=0}^{L-1} \sum_{j=0}^{L-1}(\hat{h}(i, j, t)-\bar{h})^{2}}
\end{aligned}
$$

where $\bar{h}$ denotes the average surface height. Substituting eq (16) into eq (23), the expected value of $r^{2}$ can be rewritten in terms of the state covariance as follows:

$$
\left\langle r^{2}\right\rangle=\frac{1}{\pi^{2}} \sum_{\substack{m, n=0 \\ m^{2}+n^{2} \neq 0}}^{L / 2} \sum_{p=1}^{4}\left\langle z_{p, m, n}^{2}\right\rangle
$$

The rms slope is defined as the root mean square of the slope of the surface height:

$$
\begin{aligned}
m(t) & =\sqrt{\frac{1}{\pi^{2}} \int_{0}^{\pi} \int_{0}^{\pi}\left(\frac{\partial h}{\partial x}(x, y, t)\right)^{2} d x d y} \\
& \approx \sqrt{\frac{1}{\pi^{2}} \sum_{i=0}^{L-1} \sum_{j=0}^{L-1}\left(\frac{\hat{h}(i+1, j, t)-\hat{h}(i, j, t)}{\Delta x}\right)^{2} \frac{\pi^{2}}{L^{2}}}
\end{aligned}
$$

The expected rms slope square can also be expressed in terms of the state covariance as follows:

$$
\left\langle m^{2}\right\rangle=\sum_{\substack{m, n=0 \\ m^{2}+n^{2} \neq 0}}^{L / 2} \sum_{p=1}^{4}\left(K_{p, m, n}\left\langle z_{p, m, n}^{2}\right\rangle\right)
$$

where $K_{p, m, n}$ can be computed by

$$
\begin{aligned}
K_{p, m, n} & =\frac{1}{\pi^{2}} \sum_{i=0}^{L-1} \sum_{j=0}^{L-1}\left(\phi_{p, m, n}(i+1, j)-\phi_{p, m, n}(i, j)\right)^{2} \\
& =\frac{4}{\pi^{2}} \sin ^{2}\left(\frac{\pi m}{L}\right)
\end{aligned}
$$




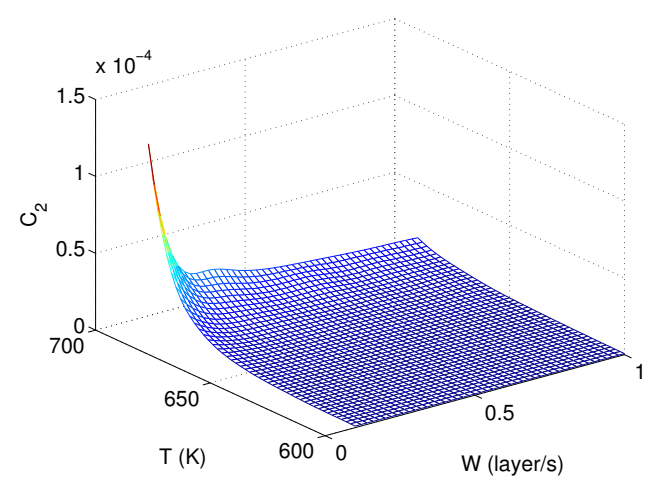

Fig. 2. $\quad c_{2}$ as a function of $T$ and $W$.

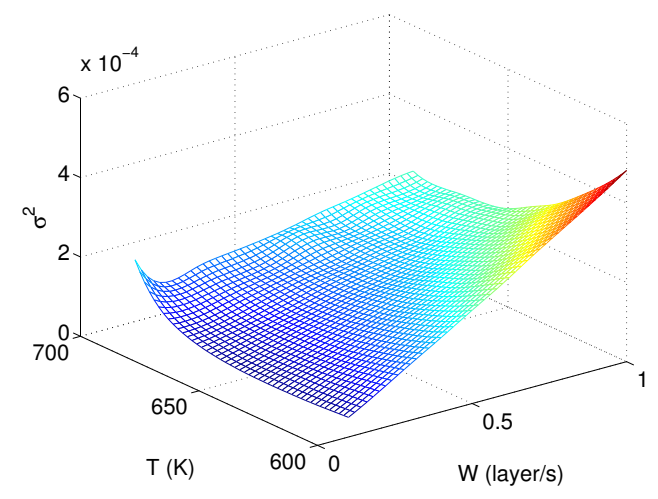

Fig. 3. $\sigma^{2}$ as a function of $T$ and $W$.

\section{Determination of $c_{2}$ and $\sigma^{2}$ as functions of $T$ and $W$}

The dependence of $c_{2}$ and $\sigma^{2}$ on substrate temperature $T$ and deposition rate $W$ can be obtained from either experiments or kinetic Monte Carlo simulations of the thin film growth process. In this work, we first carry out 100 open-loop simulations at 10 different temperatures $(T=$ $600,610, \ldots, 690 \mathrm{~K})$ and 10 different deposition rate conditions $(W=0.1,0.2, \ldots, 1.0$ layer/s). Each simulation is repeated 100 times. The simulation time is 200 seconds. Then, we determine $c_{2}$ and $\sigma^{2}$ by fitting eq. (24) and (20) to the evolution profile of $\left\langle r^{2}(t)\right\rangle$ from the obtained open-loop simulations using least-squares. Subsequently, $c_{2}$ and $\sigma^{2}$ are expressed as cubic spline functions (continuous in function value and first derivative) of $T$ and $W$. Fig. 2 and Fig. 3 show the dependence on $T$ and $W$ of $c_{2}(T, W)$ and $\sigma^{2}(T, W)$.

Because the value of $c$ affects neither $\left\langle r^{2}\right\rangle$ nor $\left\langle m^{2}\right\rangle$, it is not included in the controller formulation (please see eq. (28) below), thus no fitting was done to $c$.

\section{Multivariable Predictive Controller Design}

In this section, a model predictive controller is developed based on the dynamic model of the expected roughness square and rms slope square. Substrate temperature and deposition rate are used as the manipulated variables. The control objective is to minimize the deviation of the expected roughness square and/or rms slope square from desired setpoint values. Because the thin film deposition process is a batch process, the interval between current time and the end of the batch run is used as the prediction horizon. During each predictive controller evaluation, the manipulated variable is assumed to stay fixed until the end of the batch. To account for practical considerations, two types of input constrains are imposed. First, both the temperature and the deposition rate have lower and upper limits; second, the rates of change of both inputs are constrained to be less than certain upper limits due to actuator limitations. The resulting MPC formulation is as follows:

$$
\min _{T, W} J(t)=q_{r^{2}}\left(\left\langle r^{2}\left(t_{f}\right)\right\rangle-r_{\mathrm{set}}^{2}\right)^{2}+q_{m^{2}}\left(\left\langle m^{2}\left(t_{f}\right)\right\rangle-m_{\mathrm{set}}^{2}\right)^{2}
$$

where

$$
\begin{aligned}
& \left\langle r^{2}\left(t_{f}\right)\right\rangle=\frac{1}{\pi^{2}} \sum_{\substack{m, n=0 \\
m^{2}+n^{2} \neq 0}}^{L / 2} \sum_{p=1}^{4}\left\langle z_{p, m, n}^{2}\right\rangle \\
& \left\langle m^{2}\left(t_{f}\right)\right\rangle=\sum_{\substack{m, n=0 \\
m^{2}+n^{2} \neq 0}}^{L / 2} \sum_{p=1}^{4} K_{p, m, n}\left\langle z_{p, m, n}^{2}\left(t_{f}\right)\right\rangle \\
& \operatorname{cov}\left(z_{p, m, n}\left(t_{f}\right)\right)=e^{-8 c_{2}\left(m^{2}+n^{2}\right)\left(t_{f}-t\right)} \operatorname{cov}\left(z_{p, m, n}(t)\right) \\
& +\sigma^{2} \frac{e^{-8 c_{2}\left(m^{2}+n^{2}\right)\left(t_{f}-t\right)}-1}{2 \lambda_{m, n}} \\
& m^{2}+n^{2} \neq 0
\end{aligned}
$$

subject to:

$$
\begin{array}{cl}
T_{\min } \leq T \leq T_{\max }, & |T(t)-T(t-d t)| \leq \Delta T_{\max }, \\
W_{\min } \leq W \leq W_{\max }, & |W(t)-W(t-d t)| \leq \Delta W_{\max }
\end{array}
$$

where $t_{f}$ is the final time of the batch run, $r_{\text {set }}^{2}$ and $m_{\text {set }}^{2}$ are the respective set-points for the surface roughness square and the mean slope square, $q_{r^{2}}$ and $q_{m^{2}}$ are the weighting factors for the deviations of $\left\langle r^{2}\right\rangle$ and $\left\langle m^{2}\right\rangle$ from their set-points, $r_{\text {set }}^{2}$ and $m_{\text {set }}^{2}$ respectively, at $t_{f}, d t$ is the time interval between two successive sampling times and control actions, $T_{\min }$ and $T_{\max }$ are the lower and upper bounds on the substrate temperature, respectively, $\Delta T_{\max }$ is the limit on the rate of change of the substrate temperature, $W_{\min }$ and $W_{\max }$ are the lower and upper bounds on the deposition rate, respectively, and $\Delta W_{\max }$ is the limit on the rate of change of the deposition rate.

The optimization problem is solved at each sampling time once a new measurement of the surface height profile becomes available. An interior point method optimizer, IPOPT [18], is used to solve the optimization problem in the MPC formulation.

Remark 1: Referring to the design and implementation of estimation-based (output feedback) model predictive control, we note that an output feedback controller, which utilizes a Kalman-Bucy-type filter as the state estimator, was developed and used in the context of covariance control of a stochastic partial differential equation in a previous work of our group [5]. Furthermore, estimation-based control of a kinetic Monte Carlo model of a one-dimensional thin-film growth process was also studied in the context of roughness 
control [6] and porosity control [20]. The application of the proposed controller in conjunction with an estimation scheme will be addressed in a future work.

\section{Simulation Results}

In this section, the model predictive controller of eq. (28) is applied to the two-dimensional KMC process model. The variation of substrate temperature is from 600 to $750 \mathrm{~K}$ and the variation of the deposition rate is from 0.1 to 1 layer/s. The initial temperature is $610 \mathrm{~K}$, and the initial deposition rate is 1 layer/s. The maximum rate of change of the inputs are $\Delta T_{\max }=5 \mathrm{~K} / \mathrm{s}$ for temperature and $\Delta W_{\max }=0.05$ layer $/ \mathrm{s}$ for deposition rate. The sampling time is $5 \mathrm{~s}$. Each closedloop simulation lasts for $100 \mathrm{~s}\left(t_{f}=100 \mathrm{~s}\right)$. Expected values are calculated from 100 independent runs.

\section{A. Control of Film Surface Roughness}

First, the problem of regulating film surface roughness is considered. In this scenario, the cost function only contains penalty on the deviation of the expected surface roughness square from the set-point. The weighting factors are $q_{r^{2}}=1$ and $q_{m^{2}}=0$. The set-point is $r_{\text {set }}^{2}=3$.

Fig. 4 shows the profile of $\left\langle r^{2}\right\rangle$ under the model predictive controller of eq. (28). It can be seen that the controller drives the expected film roughness at the end of the simulation close to the desired value. Fig. 5 shows the expected profiles of $T$ and $W$ for the closed-loop simulation. Fig. 6 shows the histogram of $r^{2}$ from closed-loop simulations. We can see that $\left\langle r^{2}\right\rangle$ is close to the set-point value.

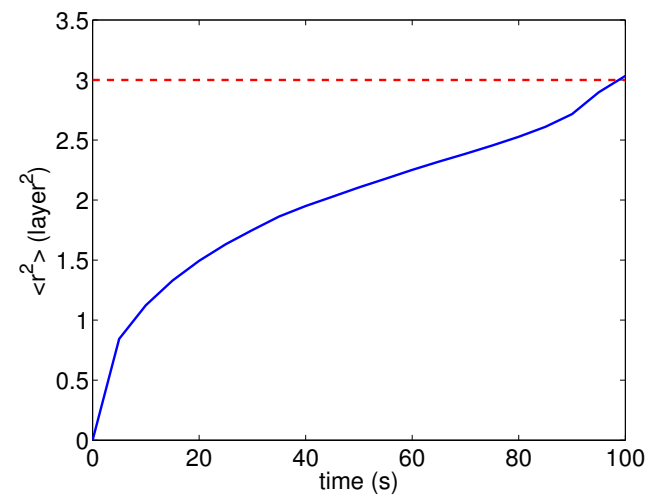

Fig. 4. Profile of expected film surface roughness square from 100 closedloop simulations. $q_{r^{2}}=1, q_{m^{2}}=0$ and $r_{\text {set }}^{2}=3$.

\section{B. Control of Film Surface rms Slope}

Next, we consider the regulation of thin film surface rms slope. The cost function includes only penalty on the deviation of the expected value of rms slope square from the set-point by choosing weighting factors $q_{r^{2}}=0$ and $q_{m^{2}}=1$. The set-point is $m_{\text {set }}^{2}=10.0$.

Fig. 7 shows the profile of expected rms slope square from 100 repeats of closed-loop simulations. The rms slope reaches its set-point at $t=100 \mathrm{~s}$. Fig. 8 shows the profile of expected temperature and deposition rate. The histogram of

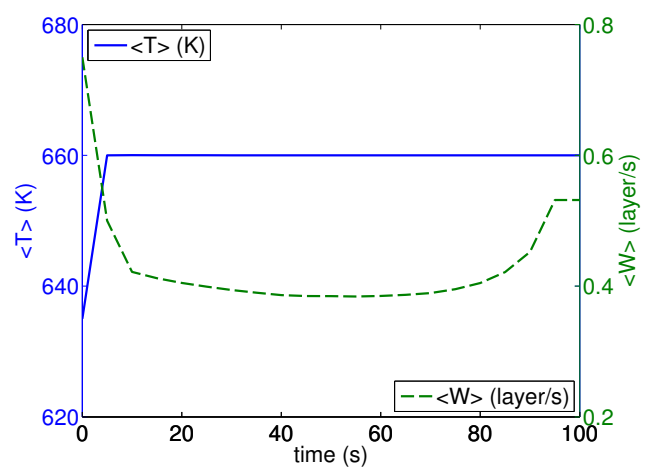

Fig. 5. Profiles of expected manipulated inputs. $q_{r^{2}}=1, q_{m^{2}}=0$ and $r_{\text {set }}^{2}=3$.



Fig. 6. Histograms of $r^{2}$ at the end of closed-loop simulation. $q_{r^{2}}=1$, $q_{m^{2}}=0$ and $r_{\text {set }}^{2}=3$.

$m^{2}$ at the end of the closed-loop simulation is presented in Fig. 9. Again, we can see that the mean value of $m^{2}$ is close to the set-point value.

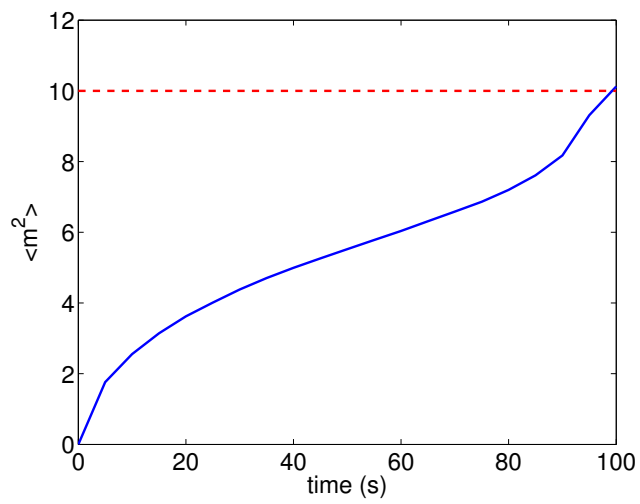

Fig. 7. Profile of expected film surface rms slope square from 100 closedloop simulations. $q_{r^{2}}=0, q_{m^{2}}=1$, and $m_{\text {set }}^{2}=10.0$.

\section{Simultaneous Control of Roughness and rms Slope}

Finally, simultaneous regulation of roughness and rms slope is carried out. The set-points of the surface roughness square and of the mean slope square are $r_{\text {set }}^{2}=3.0$ and 


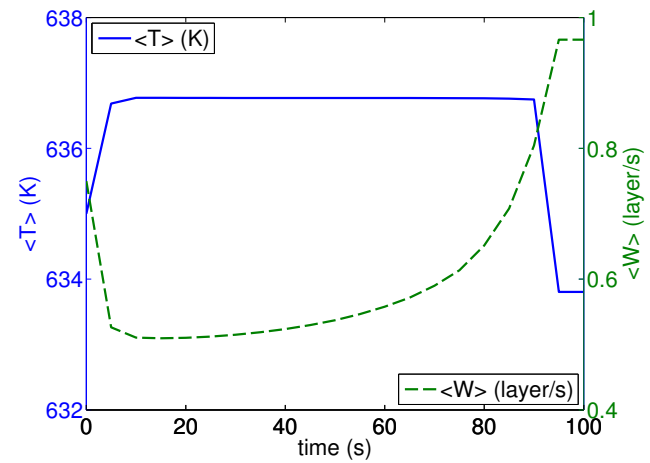

Fig. 8. Profiles of expected manipulated inputs. $q_{r^{2}}=0, q_{m^{2}}=1$, and $m_{\text {set }}^{2}=10.0$.

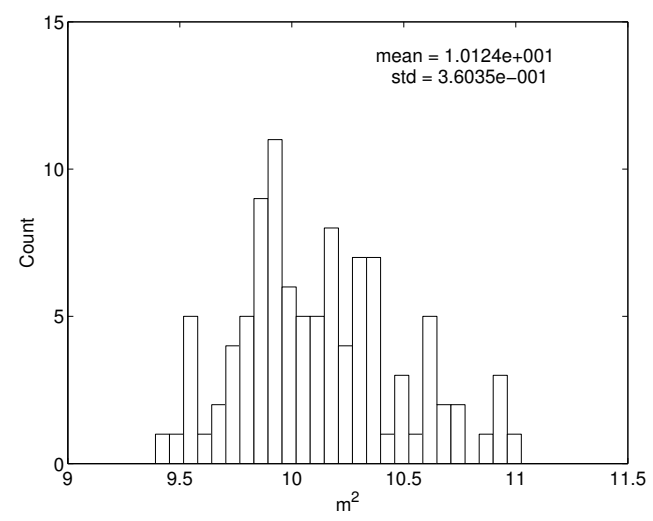

Fig. 9. Histograms of $m^{2}$ at the end of closed-loop simulation. $q_{r^{2}}=0$, $q_{m^{2}}=1$, and $m_{\text {set }}^{2}=10.0$.

$m_{\text {set }}^{2}=10.0$. The weighting factor of mean slope square is kept at 1, while the weighting factor of roughness square increases from $10^{-3}$ to $10^{5}$.

Fig. 10 shows the change of $\left\langle r^{2}\right\rangle$ and $\left\langle m^{2}\right\rangle$ as a function of $q_{r^{2}} / q_{m^{2}}$. It can be seen that as the weighting on roughness square increases, the expected roughness square approaches more closely its set-point value at the cost of larger deviation of rms slope square from its set-point value.

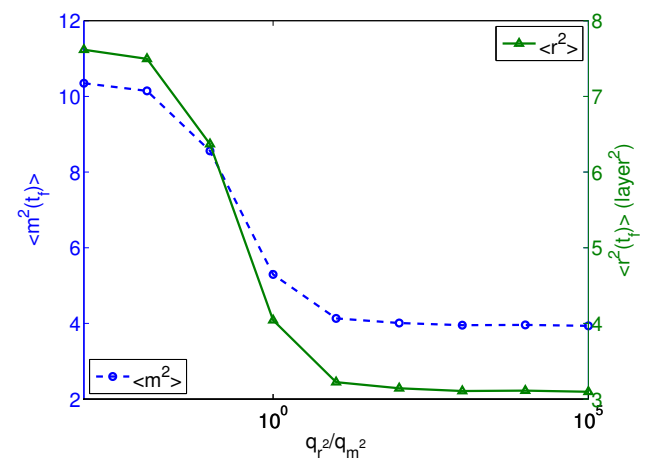

Fig. 10. $\left\langle r^{2}\right\rangle$ and $\left\langle m^{2}\right\rangle$ at the end of closed-loop simulations ( $t=100 \mathrm{~s}$ ) for different penalty weighting factors: $q_{m^{2}}=1$ and $10^{-3} \leq q_{r^{2}} \leq 10^{5}$.

\section{REFERENCES}

[1] P. D. Christofides, A. Armaou, Y. Lou, and A. Varshney. Control and Optimization of Multiscale Process Systems. Birkhäuser, Boston, 2008.

[2] S. F. Edwards and D. R. Wilkinson. The surface statistics of a granular aggregate. Proceedings of the Royal Society of London Series A Mathematical Physical and Engineering Sciences, 381:17-31, 1982.

[3] F. Family. Scaling of rough surfaces: effects of surface diffusion. Journal of Physics A: Mathematical and General, 19:L441-L446, 1986.

[4] G. Hu, J. Huang, G. Orkoulas, and P. D. Christofides. Investigation of film surface roughness and porosity dependence on lattice size in a porous thin film deposition process. Physical Review E, 80:041122, 2009.

[5] G. Hu, Y. Lou, and P. D. Christofides. Dynamic output feedback covariance control of stochastic dissipative partial differential equations. Chemical Engineering Science, 63:4531-4542, 2008.

[6] G. Hu, Y. Lou, and P. D. Christofides. Model parameter estimation and feedback control of surface roughness in a sputtering process. Chemical Engineering Science, 63:1810-1816, 2008.

[7] G. Hu, G. Orkoulas, and P. D. Christofides. Modeling and control of film porosity in thin film deposition. Chemical Engineering Science, 64:3668-3682, 2009.

[8] G. Hu, G. Orkoulas, and P. D. Christofides. Regulation of film thickness, surface roughness and porosity in thin film growth using deposition rate. Chemical Engineering Science, 48:3903-3913, 2009.

[9] J. Huang, G. Hu, G. Orkoulas and P. D. Christofides. Dynamics and Lattice-Size Dependence of Surface Mean Slope in Thin Film Deposition. Industrial \& Engineering Chemistry Research, 50:12191230, 2011.

[10] J. Müller, B. Rech, J. Springer, and M. Vanecek. TCO and light trapping in silicon thin film solar cells. Solar Energy, 77:917-930, 2004.

[11] D. Ni and P. D. Christofides. Multivariable predictive control of thin film deposition using a stochastic PDE model. Industrial \& Engineering Chemistry Research, 44:2416-2427, 2005.

[12] A. Poruba and A. Fejfar. Optical absorption and light scattering in microcrystalline silicon thin films and solar cells. Journal of Applied Physics, 88:148-160, 2000.

[13] S. F. Rowlands, J. Livingstone, and C. P. Lund. Optical modelling of thin film solar cells with textured interfaces using the effective medium approximation. Solar Energy, 76:301-307, 2004.

[14] J. Springer and A. Poruba. Improved three-dimensional optical model for thin-film silicon solar cells. Journal of Applied Physics, 96:53295337, 2004.

[15] A. Varshney and A. Armaou. Multiscale optimization using hybrid PDE/kMC process systems with application to thin film growth. Chemical Engineering Science, 60:6780-6794, 2005.

[16] A. Varshney and A. Armaou. Reduced order modeling and dynamic optimization of multiscale pde/ $\mathrm{kmc}$ process systems. Computers \& Chemical Engineering, 32:2136-2143, 2008.

[17] D. G. Vlachos, L. D. Schmidt, and R. Aris. Kinetics of faceting of crystals in growth, etching, and equilibrium. Physical Review B, 47:4896-4909, 1993.

[18] A. Wächter and L.T. Biegler. On the implementation of an interiorpoint filter line-search algorithm for large-scale nonlinear programming. Mathematical Programming, 106(1):25-57, 2006.

[19] M. Zeman and R. Vanswaaij. Optical modeling of a-si : H solar cells with rough interfaces: Effect of back contact and interface roughness. Journal of Applied Physics, 88:6436-6443, 2000.

[20] X. Zhang, G. Hu, G. Orkoulas, and P. D. Christofides. Controller and estimator design for regulation of film thickness, surface roughness and porosity in a multiscale thin film growth process. Industrial \& Engineering Chemistry Research, 49:7795-7806, 2010.

[21] X. Zhang, G. Hu, G. Orkoulas, and P. D. Christofides. Multivariable model predictive control of thin film surface roughness and slope for light trapping optimization. Industrial \& Engineering Chemistry Research, 65:6101-6111, 2010.

[22] X. Zhang, G. Hu, G. Orkoulas, and P. D. Christofides. Predictive control of surface mean slope and roughness in a thin film deposition process. Chemical Engineering Science, 65:4720-4731, 2010. 